$\frac{30}{310146}$
De\# 04/1-1

UCID- 21140

\title{
Preparing the Alcator C Bolometer System for Use on MTX
}

\author{
Marty Marinak \\ University of California \\ Berkeley, California
}

February 9,1988

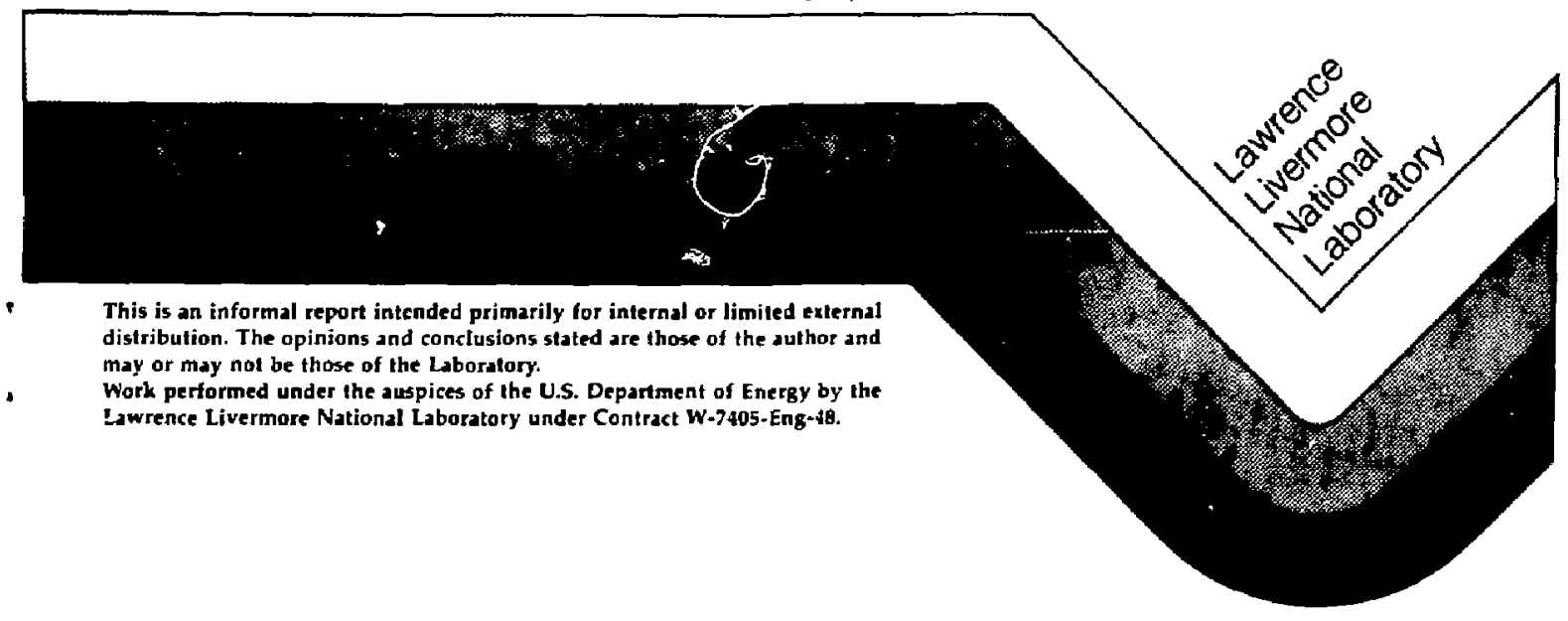




\title{
ABSTRACT
}

The Alcator $C$ bolometer array has been modified to be compatible with electron cyclotron heating on the Microwave Tokanak Experiment. Fine wire mesh screens are mounted on the front of the bolometer collinator tubes to attenuate microwave heating of the boloneters. Structural changes eliminate openings in the seans of the bolometer housing, which represent athways for microwaves to enter the system. This paper outlines the operatio.al principles of the belometer system, discusses the measured and predicted perfomance characteristics of the bolometer array, and includes a zoncise guide to the operation of the bolometer controller.

UCID - 21147

DE88 00672

\section{DISCLAIMER}

\begin{abstract}
This report was prepared ax an mcooupl of work sponsored by an agency of the United Statea Government. Neither the United States Goweroment bor any agency thereor, nor any of their employes, makes any warranty, express or implied, or assumes any legal liability or responsibility for the accuracy, completenes, or usefulness of any infoniation, apparatus product. or procese discloced, or represeats that ies use would axe infringe privately owned rights. Refer. ence berein to any specific commercial product, prosest, or service by trade aame, trademark, manufacturer, or otherwixe docs not necesurily contititute or imply its endorsement, repornmendation, of favoring by the Uaited States Government or any agency thereor. The views and opinions of authors expreand berein do not necessarily state of reflect thoue of the United State Government or any arency thereof.
\end{abstract}


Contents

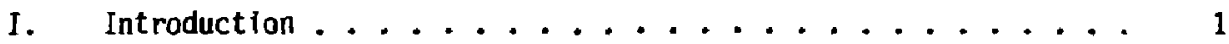

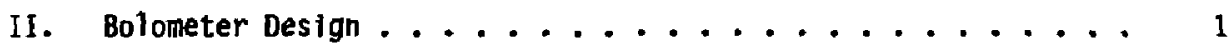

III. Bolometer Measurement arid Control Circuitry ....... 2

IV. Magnetic Field Interaction .............. 3

V. Assessment of Contributions to Boloneter Heating ....... 3

VI. Assessment of the Longevity of the Screens ......... 7

VII. Operation of the Boloneter Controller .......... 8

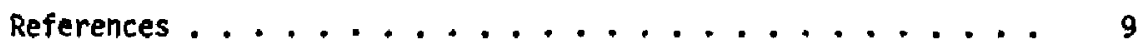

Appendix: Tests of System Performance .......... 10

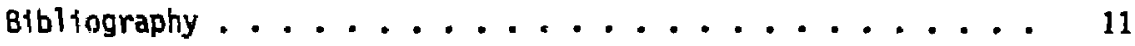




\section{INTRODUCTION}

Bolometers are used to measure the total power radiated from a fusion plasma. On MTX we use the Alcator $C$ bolometer system, comprised of an array of 16 bolometers with collimator tubes that allow viewing of different radial segments of the plasma. The array must be mounted on a top port, preferably as far away from the free electron laser (FEL) as possible. The Alcator $C$ bolometer system was not designed to be compatible with microwave heating schemes. The 1.2- $\mathrm{mm}$ waves from the FEL would heat the bolometers, giving false signals: the bolometer electronics might amplify microwave signals absorbed in the bolometer circuitry if corrective modifications are not made.

To shieid the bolometers from microwave energy, we chose fine, electroformed copper meshes at a spacing equivalent to $750 \mathrm{wires} / \mathrm{in}$. The copper meshes are mounted over the front of the bolometer collimator tubes. Tests on similar meshes indicate that they will attenuate the microwave signal penetrating into the bolometer tubes enough that FEL heating of the bolometers should be nearly indlscernible above the bolometer circults' electronic noise. Tests on the system also indicate this amount of microwave energy inside the bolometer produces no measurable increase in noise in the bolometer circuitry. other physical modifications made on the bolometer system prevent microwaves from entering through seams and openings in the structure.

The bolometer controller is the same device used on the bolometers on the Tandem Mirror Experiment. The controller was designed at Princeton to re used with 50-1 platinum wire bolometer pairs and is completely compatible with the Alcator $C$ bolometer array. Malfunctioning bolometer controller circuits are repaired and aligned with the individual bolometer cells.

\section{BOLOMETER DESIGN}

Observations of the MTX bolometers indicate that they are the most recent version developed for the tokanak fusion test reactor. The sensor consists of platinum wire sandwiched between layers of epoxy and platinum foil. The active sensor is cemented at the ends of a copper heat sink. A reference sensor is mounted on the back of the heat sink. Shielded from the plasma, its entire area is cemented to the heat sink to remain in thermal contact. The copper heat sink is covered with a film of insulating material, likely epoxy. 
Platinum sensors are used because they can withstand temperatures of up to $200^{\circ} \mathrm{C}$, are highly resistant to damage by neutron and gamma radiation, and have a minimal outgassing. In addition, the platinum has a nearly constant temperatuma resistance coefficient over the bolometer temperature operating range.

\section{BOLOMETER MEASUREMENT AND CONTROL CIRCUITRY}

The active and reference sensors and fixed resistors in the electronics chassis are connected to form a wheatstone bridge. The bridge is excited with a square wave at $20 \mathrm{kHz}$ to reject pick-up voltages induced by the changing electromagnetic fields of the tokamak. To eliminate the need for a reactance acjustment, the synchronous detector is gated off for a time after each reversal of the square wave. The contral electronics establish a reference power input to the bolometer sensors.

Two modes of operations are possible. In the open loop (or temperature) mode, the bridge is balanced at the time of the "t-zero" pulse. Changes in the incident radiation intensity change the temperature of the active sensor. The resistance change induces a voltage across the bridge. To determine the absorbed power versus time in this mode, it is necessary to unfold a detector cooling curve from the measured sensor temperature versus time. On TMX this mode was used most often because electronic noise (likely from the neutral beams) was present in the bolometer circuit, and the operators felt they could discriminate the noise from the data better when there was minimum processing of the signal data between the input and output of the controller circuitry.

Another mode of operation is avai,able, however, lacking some limitations of the conventional mode of operation. In this closed loop (or power) mode, the sensor is maintained at constant temperature by modulating the electrical power dissipated in the sensor. This prov.jes a direct measure of the absorbed power from the plasma. In this mode there is an output proportional to absorbed power, and the result is independent of the sensor thermal capacitance and resistance for our bolometer systen. Besides simplifyiry calibration and data analysis, this mode of operation has an inherently rapid time constant depending primarily on the ratio of the cfrcuit gain to the sensor thermal capacitance. 
A built-in feature of the circuit is an electrical power pulse to calibrate the detector. A dc offset is added to the square wave applied to the bridge. Only the active sensor sustains a significant resistance increase due to the increased power. This electrical callbration can be used in either mode.

\section{MAGNETIC FIELD INTERACTION}

Platinum wires function as strain gauges. The alternating $\vec{I} \times \vec{B}$ force on the strands of the sensor grid can excite vibrations which would produce noise in the output signal. The bolometer grids are cemented to a backing of kapton and platinum in order to reduce this effect. Furthermore, all bolometers are mounted so that strands are parallel to the toroidal field.

\section{ASSESSMENT OF THE CONTRIBUTIONS TO BOLOMETER HEATING}

The majority of the radiation is expected to be in the far ultraviolet and soft $x$ ray. The bolometer collimator tubes have a threaded, blackened inner surface, which improves colltimation of visible light and actually helps 1.2-mm microwaves to propagate down the tube. Measurements of the angular discrimination of the bolometer collimator tubes show that they have a steep cutoff with a half maximum value when the incident line radiation is at $2^{\circ}$ to the axis of the tube (Fig. 1). The platinum foil covering the bolometers is somewhat reflecting in the uitraviolet region and about $50 \%$ reflecting in the visible region. The copper meshes to be placed at the front of the collimator tubes should transmit $55 \%$ of the incident visibie and uitraviolet radiation at normal incidence. When mounted on the bolometer, the mesh will be deliberately slackened to allow it to fit over the collimator tubes. These meshes are easily torn and thus cannot withstand much tension. one should avoid touching them and take suitable care when installing the bolometer in its port. The wire mesh screens have a negligible effect on the angular discrimination of the collimating tubes $(F i g .2 a, b) . X$ rays of energies greater than $3 \mathrm{keV}$ will pass through the wires in the mesh to some extent. For a copper mesh $6 \mu \mathrm{m}$ thick, $x$ rays with an energy of 4.51 keV will pas 5 through the fraction of screen area occupjed by the wires at a rate of $18.6 \%$ for wires with a rectangular cross section such as ours. For these screens 
the transmission of $x$ rays of energy lass than 3 keV through the wires is negligible; the attenuation of the lower-energy $x$ rays by the screens is the same as that for visible light.

The reflectivity of platinum to various wavelengths is listed in Table 1 and shown in Fig. 3. The platinum foll covering the sensor is excellent for absorbing $x$ radiation. All soft $x$ rays incident upon the sensor are absorbed by $i t$. The mean-free path of $x$ rays in platinum becomes comparable to the foil thickness at an energy of approximaiely $16 \mathrm{keV}$. A compilation of the sensitivity of the bolometers to electromanetic radiation at different energies is given in Fig. 4. This takes into account the reflectivity of platinum and the mass absorption coefficients of copper and platinum for photons of various energies. Observation of the screens with a microscope at $1000 x$ magnification reveals that the wires are flat on one side and slightly rounded on the other, giving them a nearly unfform depth across. According to the manufacturer, the wire depth is nominaliy $1.5 \times 10^{-4}$ in. The piatinum foll across the top of the bolometer is nominally $3.5 \times 10^{-4} \mathrm{~cm}$ thick. The latter numbers were used to generate fig. 4 .

In addition to electromagnetic radtation from visible through $x$ ray, which we intend this instrument to measure, there will be contributions to bolometer-neasured energy from FEL radiation, synchrotran radiation, infrared radiation (from the limiters), neutron and gamma radiation, and neutral atoms. The center collimator tubes will be nearly flush with the edge of the tokamak outer wall. The flux of charged particles into the bolometer tubes should be negligible because the bolometers are far enough behind the limiters.

\section{A. HEATING BY NEUTRAL PARTICLES}

The heating of the collimator tubes by neutral particles should be negligible. The solid angle viewed by the bolometers is $3.8 \mathrm{mSr}$. For a flux of $1.0 \times 10^{14}$ neutrals $/ \mathrm{cm}^{2} \mathrm{~s}$ at $1 \mathrm{keV}$, the bolometer would see $13 \mathrm{pw}$ of power, including attenuation by the screens. This would be difficult to distinguish from circuit noise. It is felt that the estimate of $1.0 \times 10^{14}$ neutrals $/ \mathrm{cm}^{2} \mathrm{~s}$ is rather conservative. 


\section{B. NEUTRON HEATING}

For D-D (deuterium) shots the neutron heating of the bolometers should be at least an order of magnitude lower than the impurity radiation. To separate the neutron and gamma flux contributions to bolometer heating, one could cover a bolometer to allow only neutrons and gammas to pass through.

\section{INFRARED AND MICROWAVE HEATING}

The bolameter has been located at the top $F$ port to minimize the microwave energy flux and infrared radiation flux incident upon it. The theory for a thin, inductive mesh predicts that our 750 lines/in. screens should attenuate normally incident microwave radiation by more than $35 \mathrm{~dB}$. Microwave transmission tests were performed on two nickle meshes of 250 and 400 LPI at a frequency of $140 \mathrm{GHz}$. For the 250 -LPI screen, the measured ratio of transmitted to incident-normal radiation was $-16.9 \mathrm{~dB}$. When the mesh was allowed to hang wrinkled on the mounting frame (as our final screens will), the ratio dropped to $-21.4 \mathrm{~dB}$. This drop is thought to be partially due to the greater reflectivity of the mesh when the radiation is incident at an angle with the normal and partially due to scattering of transmitted microwaves out of the path between the source and receiver horns used in the test. With the 400-LPI screen, the attenuation at normal incidence was measured at $-19.1 \mathrm{~dB}$. With a "wrinkled" screen the attentuation was measured at $-22.7 \mathrm{~dB}$. There is a large difference between the neasured performance of these screens and the prediction of the theory for thin, nonresistive screens. The large difference between the predicted and measured screen performance is due partly to the surface resistivity of the metal but mostly due to the nonnegligible thickness of these wire mesh screens. Thin meshes made of perfectly conducting material obey the relations:

$$
\begin{aligned}
& \tau=1+\Gamma \\
& |\tau|^{2}+|\Gamma|^{2}=1,
\end{aligned}
$$

where $\tau$ and $\Gamma$ are the transmission and reflection coefficients. For an incident wave of unit amplitude, $|\Gamma|$ is the magnitude of the scattered wave. 
The points satsifying these relations form a circle (Fig. 4). The resistivity of the screen would cause a shift in the locus of these points in the direction of the real axis. The magnitude of the effect of resistivity can account for only a tiny fraction of the discrepancy observed. A theoretical treatment by Marcuvitz ${ }^{1}$ predicts that the finite thickness of the mesh shifts the $\Gamma$ curve in the negative imaginary direction and the 1 - $\tau$ curve equally in the positive Imaginary direction. Experimental observations by Ulrich ${ }^{2}$ and others confirm this shift. The absolute magnitude of the shift is, however, considerably larger than the theory predicts. This might be an inherent difference between two-dimensional and one-dimensional meshes. In all theories covering inductive and capacitive meshes, the parameters of interest are the ratios of mesh spacing to wavelength, wire/plate width to spacing, and wire/plate thickness to spacing. The 400-LPI mesh was chosen to simulate at $140 \mathrm{GHz}$ as closely as possible the performance of a 750 -LPI mesh at $250 \mathrm{GHz}$. Published experimental results indicate that copper meshes are better attenuators of millimeter microwaves than nicke] meshes, by more than the difference for which bulk resistivities alone would account. Therefore, with the method of mounting planned, we can expect to achieve $22.7 \mathrm{~dB}$ of attenuation for the 1.2-mon waves with 750-LPI copper screens.

A test was conducted on the bolometer to measure the sensor absorption of microwaves. All openings out of the bolometer were covered, and power refiected into the waveguide horn was measured. When a directional horn was used to launch power into a tube $0.9 \%$ of the net input energy (at $140 \mathrm{GHz}$ ) was absorbed by the sensor. When an open-ended waveguide was used 0.6-0.7\% was absorbed. Microwaves launched from a directional horn outside the tube normally incident were absorbed at a rate of $0.4-0.5 \%$.

Based on preliminary data from the measurement of the $Q$ of the Alcator $C$ cavity at $140 \mathrm{GHz}$, one expects that for a power input of $2 \mathrm{MW}$ of microwaves into the empty cavity one would measure an average power flux of $400 \mathrm{~W} / \mathrm{cm}^{2}$ at the cavity outer wall. Including a factor for the attenuation of the microwave flux by plasma absorption, one can estimate the FEL heating of a bolometer sensor:

$$
\left(1.17 \mathrm{~cm}^{2}\right)\left(400 \mathrm{~W} / \mathrm{cm}^{2}\right)\left(A_{p l a s m a}\right)\left(10^{-2.27}\right)(0.01)<10^{-4} \mathrm{~W} A_{p l a s m a}<0.004 .
$$


Estimates of the fraction of power absorbed by the plasma on a single perpendicular pass range from 0.5 to 0.98 , the lower figure due to nontinear absorption on the first pass. Linear absorption predicts $80 \%$ absorption during startup and $98 \%$ absorption when the electron temperature reaches $1.8 \mathrm{keV}$. If $\mathrm{BO} \%$ is absorbed per pass on the average, the number of such passes needed to attenuate the microwave flux to 0.004 of the initial magnitude is

$$
\frac{\log (0.004)}{\log (0.2)}=3.4
$$

One can expect such attenuation to occur by the time microwaves have traveled to the opposite side of the torus. A signal resulting from $100 \mu \mathrm{W}$ of power should be much smaller than the impurity/line radiation. If the microwaves made just one more pass through the plasma, effectively attenuating the intensity by another order of magnitude, the signal magnitude would be buried in the noise in the closed loop mode. Because the synchrotron radiation will be much less intense, it won't contribute significantly to bolometer heating. The wire mesh screen's transmittance goes, very roughly, as the ratio of mesh spacing to radiation wavelength, so that harmonics radiated by the plasma will be effectively shielded by the screen. Thus, the screens improve the bolometer's capability to exclude microwave power from the measured power.

\section{ASSESSMENT OF THE LONGEYITY OF THE SCREENS}

The wire mesh screens should be able to withstand constant heating resulting from steady-state operation of the FEL without any plasma. The sputtering from energetic neutral particles will degrade the screens only over a very long period of time. Sputtering and heating of the screens during Taylor discharges (cleaning) will be negligible. Other phenomena, such as unipolar arcs, may shorten the life of the screens. Thus, periodic inspection of the screen, using a microscope with a magnification in the range of $500 x$, is recommended. 
The MTX bolometer controller can be issued commands through the front panel when the REMOTE/LOCAL switch is in the LOCAL position. The remote setting allows it to be configured to various states by digital electrontc comnands through a connection on the rear panel. The entire system can be tested without the use of a computer. The channel selector, which operates in base 8 , allows one to look at the output of a particular cfrcuit through the TLM OUT outlet. The LIVE setting allows one to look at the instantaneous output of a circuit while the MEM setting locks into the 200-ms memory built into the unit.

Other controls function as follows:

- MEM SYNC Synchronizes an oscilloscope with the memory output.

- EXT SYNC IN Triggers the controller into the hold balance mode when it produces data. After about $5 \mathrm{~s}$, the controller reverts to its original state.

- gain test In the open loop mode, it simulates a known temperature change in the sensor allowing calibration.

- HRT ENABLE Readies the memory to fill itself with new data at the time of the next trigger.

- PULSE ENABLE Enables the calibration electrical power pulse system.

- PWR PULSE Triggers a pulse. The HOLD BAL light is lit whenever tine controller is producing data.

- POWER MAGNITUDE Selects the magnitude of the power pulse settings: low, med, and hi correspond to $0.8,1.6$, and $3.2 \mathrm{~mW}$ nominally. The actual value of the offset voltage can be monitored through channel 67.

- PULSE WIOTH Selects the duration of the power pulse. The settings narrow, med, and wide correspond to $10 \mathrm{~ms}, 100 \mathrm{~ms}$, and 1 s.

- PULSE SYNC Sends a pulse to synchronize other equipment with the controller.

- abS PWR

Allows one to measure the reference power delivered to a sensor. The transfer function is $0.5 \mathrm{~V} / \mathrm{mW}$. 
- NB FILTER

In the wideband mode the contrcller output is passed through a 250-Hz low-pass filter. With the narrow band filter in, the output filtei is $50 \mathrm{~Hz}$. The filter is not simply a line filter but an active part of the bolometer circuitry.

- LOOP Open lo:p = temperatur:

\section{REFERENCES}

1. N. Marcuvitz, Waveguide Handbook, (McGraw-Hi11, New York, 1951).

2. R. Ulrich, "Far-Infrared Properties of Metallic Mesh and Its Complementary Structure," Infrared Physics 7, 35-55 (1967). 


\section{APPENDIX}

Summary of Tests

Closed Loop Measurements

Transfer functions

Noise
$2.50 \mathrm{~V} / \mathrm{mll}$ wideband

$2.38 \mathrm{~V} / \mathrm{m}$ H narrowband

11.0 ms wideband

14.5 ms narrowband

$20 \mathrm{mV}$ rms $=8 \mu \mathrm{W}$ widebano

$7 \mathrm{mV} \mathrm{rms}=2.8 \mathrm{jW}$ narrowband

Auto balance hold drift test

$2.8 \mu \mathrm{W} / \mathrm{s}$

Open Loop Measurements

Transfer function

C

$R$

Auto balance hold drift mode

Noise (wideband)
$20 \mathrm{~V} /{ }^{\circ} \mathrm{C}$

$8.6 \times 10^{-4} \mathrm{~J} /{ }^{\circ} \mathrm{C}$

$168^{\circ} \mathrm{C} / \mathrm{W}$

$280 \mu^{\circ} \mathrm{C} / \mathrm{s}$

$115 \mu^{\circ} \mathrm{C} / \mathrm{s} \mathrm{rms}$ 


\section{BIBLIOGRAPHY}

N. Marcuvitz, Waveguide Handbook. (McGraw-Hill, New York, 1951).

C. L. Mok, "The Far-Infrared Performance and Application of Free-Standing Grids Wound from $5 \mu \mathrm{m}$ Diameter Tungsten Wire, Infrared Physics 19, 437-442 (1979).

J. Schivell, G. Renda, J. Lowrance, and H. Hsuan, "Development of a Bolometer for Measurements on High Temperature Plasmas," Rev. Sci. Instrum. 53 , $1527-1534$ (1982).

R. Ulrich, "Far-Infrared Properties of Metallic Mesh and Its Complementary Structure," Infrared Physics 2, 35-55 (1967).

J. R. Reitz, F. J. Milford, and R. W. Christy, Foundations of Electromagnetic Theory (Addison-Wesley, Reading, MA, 1980), 3rd ed., 412-416.

K. J. Button, "Perlodic Waveguide Structures," Infrared and Mi11 imeter Waves, vol. 1 (Acadenic Press, New York, 1979) 252-259. 
Table 1. Platinum reflectivity at normal incidence for visible wavelengths.

$\begin{array}{lll}450 \mathrm{~nm} & 550 \mathrm{~nm} & 750 \mathrm{~nm} \\ 55 \% & 60-65 \% & 71 \% \\ \end{array}$




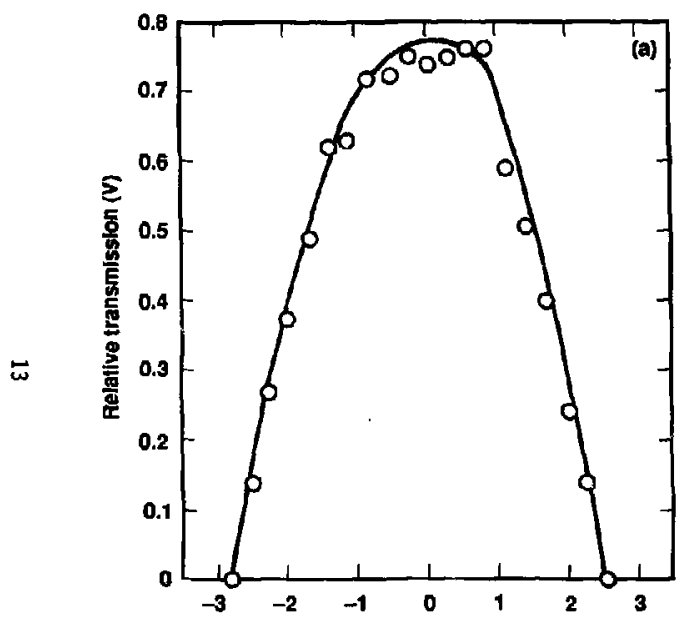

Angle of incldent light with nomal (degrees)

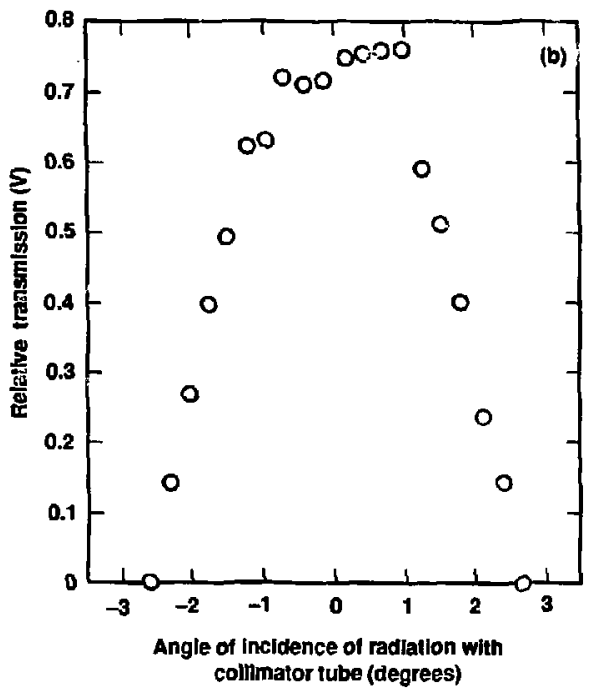

Figure 1. (a) Anguiar discrimination of bolometer collimator tubes:

(b) angular discrimination of bolometer collimator tube with respect to visible, ultraviolet, and $x$ radiation. 

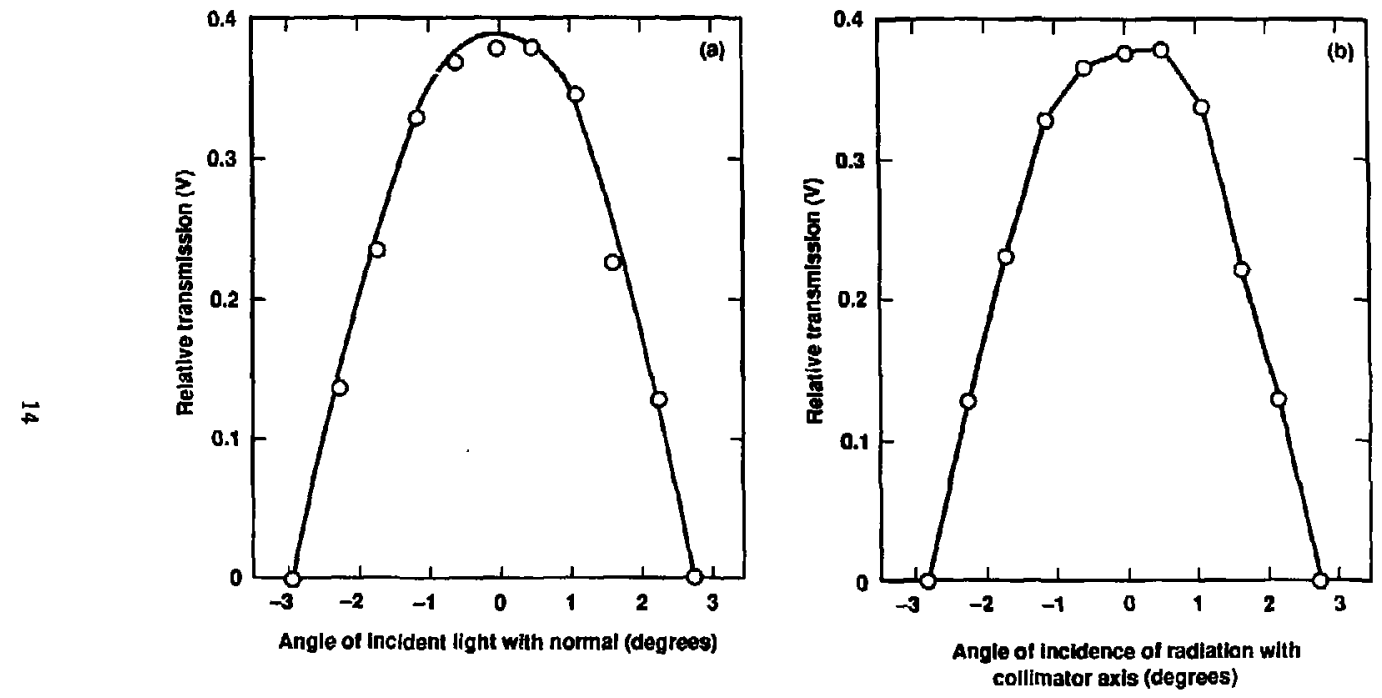

Figure 2. Angular discrimination of bolometer collimator tubes: (a) wide 400-LPI mesh (nickel); (b) with respect to visible, ultravtolet, and $x$ radiation with wire mesh screen in place. 


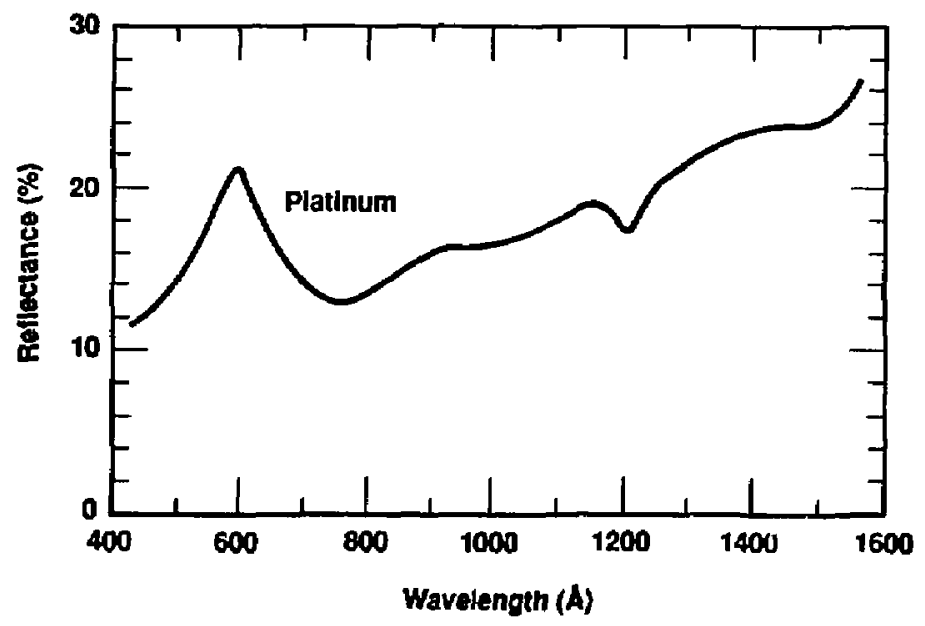

Figure 3. Platinum reflectivity at normal incidence for ultraviolet wavel engths.

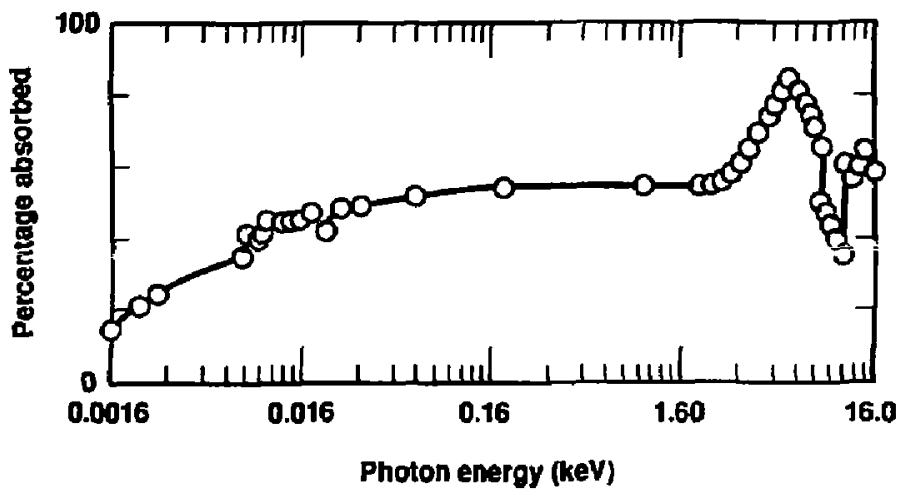

Figure 4. Relative bolometer detection efficiency. 


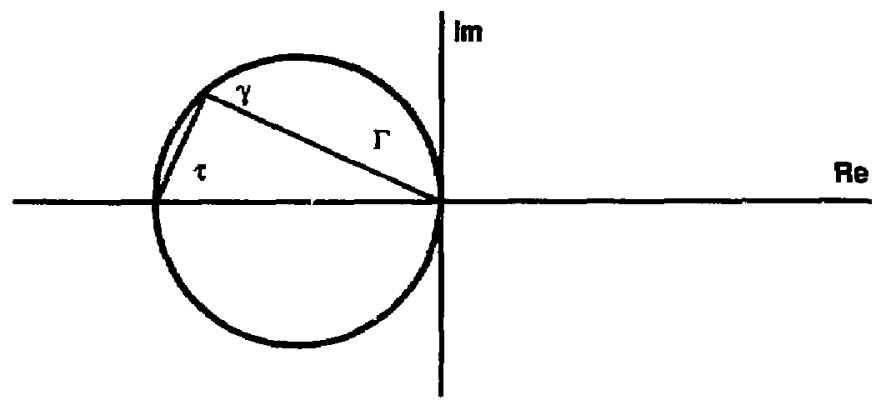

Figure 5. The transmission and reflection vectors. 